\title{
Perforated Incisional Littre's Hernia: A Rare Content of a Common Hernia
}

\author{
Kumarappan Al, Norly S, Samuel Tay, Vicknesh C \\ Department of Surgery, Hospital Pakar Sultanah Fatimah, Jalan Salleh, 84000 Muar, Johor
}

\begin{abstract}
Anticipation of complications is of paramount importance for a surgeon. Incisional hernia is a well-known complication for abdominal surgeries. Risk factors such as increasing age, obesity, male gender are well known. Intestinal obstruction, strangulation and perforation are imminent complications of this type of hernia if they become incarcerated. Common contents of a ventral incisional hernia are small bowel, large bowel and omentum. Even though Meckel's diverticulum is a common gastrointestinal anomaly, it is rarely found to be the content of a hernial sac. A hernia sac containing Meckel's diverticulum is also known as Littre's hernia. Only a few cases have been reported in the literature. Thus here we present a case of perforated Meckel's diverticulum that was entrapped in the ventral incisional hernia.
\end{abstract}

KEYWORDS: Littre's hernia, Incisional hernia, Meckel's diverticulum

\section{INTRODUCTION}

One of the complication to watch out after abdominal surgery is an incisional hernia. It can remain asymptomatic but usually produces symptoms once it gets incarcerated and can impose significant life threatening risk if it becomes strangulated or perforated.

The common contents are small bowel, large bowel and omentum. Meckel's diverticulum presenting as a content of an incisional hernia have only been reported scantily. Meckel's diverticulum is the most common gastrointestinal anomaly, reported to be present in $2 \%$ of the population. ${ }^{1}$ It is a true diverticulum whereby it is composed of all layers of the intestine. Clinically and radiologically, it is difficult to determine the content of the hernia, so the diagnosis is mainly made intra-operatively. Only $4 \%$ of people with Meckel's diverticulum end up being symptomatic whereas the rest carries them to grave unknowingly. ${ }^{1}$

A hernia sac containing Meckel's diverticulum is also known as Littre's hernia. Incisional Littre's hernia is a rare entity and may be very difficult to be diagnosed preoperatively.

Corresponding author:

Kumarappan Alagappan

Department of Surgery,

Hospital Pakar Sultanah Fatimah,

Jalan Salleh,

84000 Muar, Johor,

Malaysia.

Tel no: 00-60-16-2784688

Fax: 00-60-6-9526003

Email: alkums@hotmail.com

\section{CASE REPORT}

Our patient is an 81 year old Malay gentleman who had a laparotomy and omental patch repair for a perforated prepyloric ulcer five years ago. He was diagnosed to have an incisional hernia during an outpatient visit. The content of the hernia sac was confirmed by an ultrasonography examination to be bowel loops (Figure 1).

He was counselled for an elective incisional hernia repair whereby he refused citing his old age. Subsequently he was well till the last two months it became irreducible but he was asymptomatic.

Two days prior to admission he was having colicky central abdominal pain which progressively turned persistent. This was associated with vomiting and minimal passing of flatus and faeces. Upon examination he was noted to be dehydrated. There was a tense, irreducible swelling at the previous laparotomy scar measuring approximately $4 \times 4 \mathrm{~cm}$.

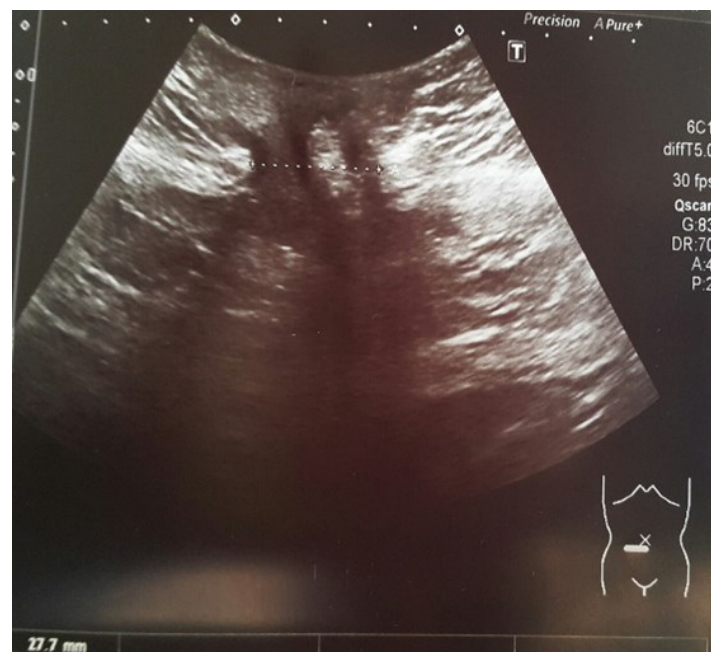

Figure 1: Ultrasonography showing the fascial defect with bowel loops in the hernia sac 
Bowel sounds were present over the swelling. Abdominal radiograph showed prominent small bowel with no pneumoperitoneum (Figure 2). Blood investigation revealed neutrophilic leukocytosis. After adequate resuscitation he was taken to operating theatre with the diagnosis of strangulated incisional hernia. Intra-operatively, Meckel's diverticulum was noted in the sac with presence of slough and minimal pus, suggestive of sealed perforation (Figure 3).
Segmental resection of the small intestine involving the Meckel's diverticulum was undertaken with end to end anastomosis. The fascial defect was closed primarily. Post-operative recovery was uneventful and he was discharged well after five days. Histopathological examination of the resected bowel confirmed it to be Meckel's diverticulum (Figure 4a \& 4b).

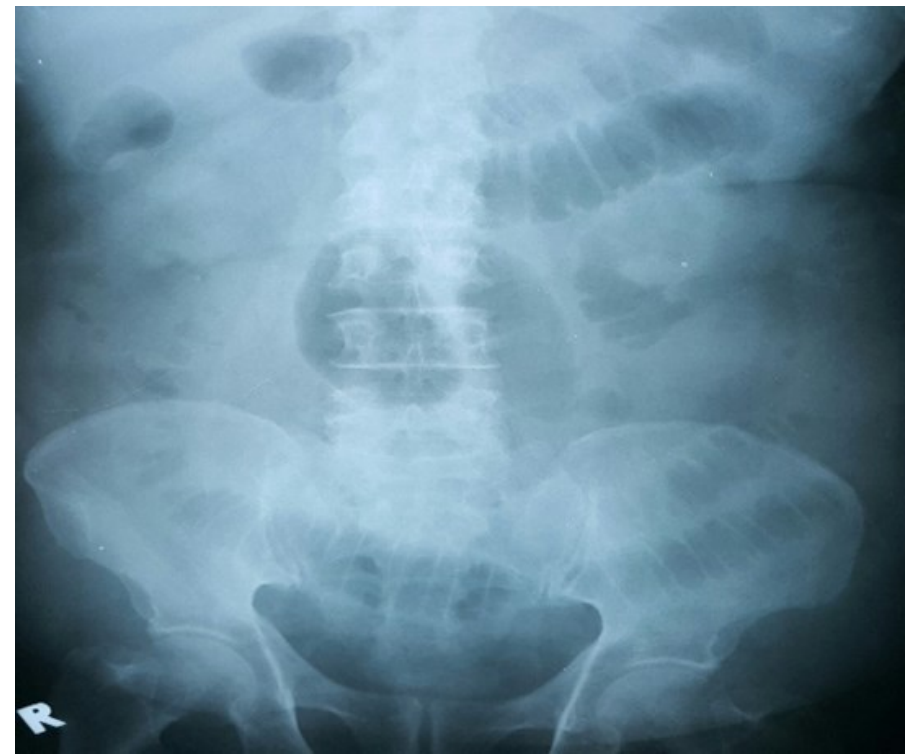

Figure 2: Abdominal radiograph showing prominent small bowel (stacked coin appearance) with no pneumoperitoneum

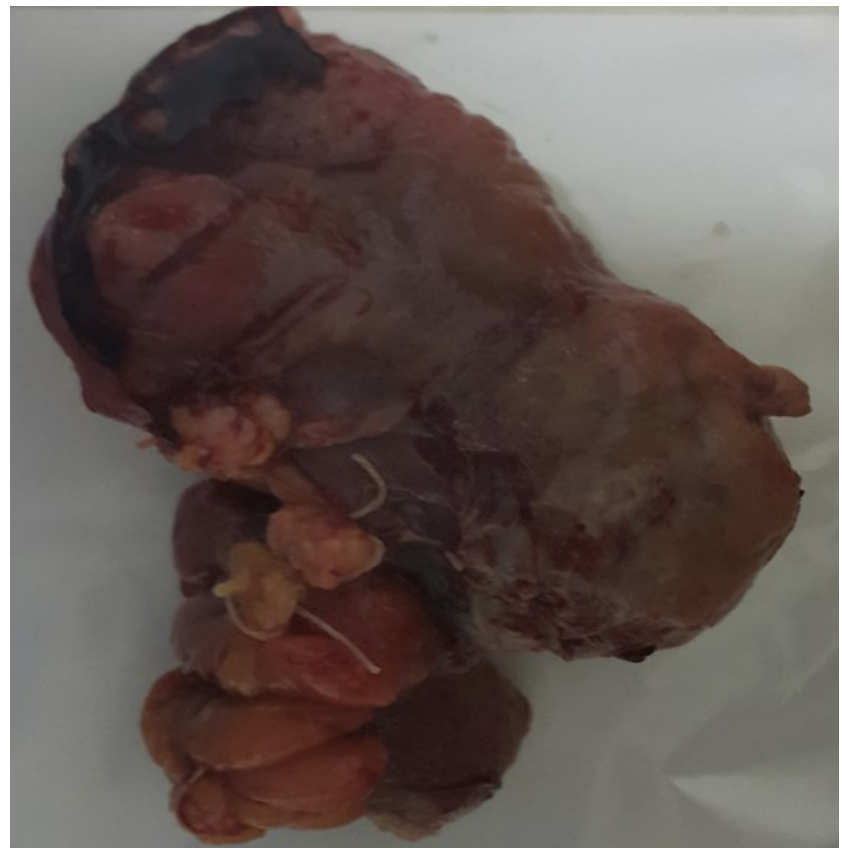

Figure 3: Outpouching of the Meckel's diverticulum covered with slough 


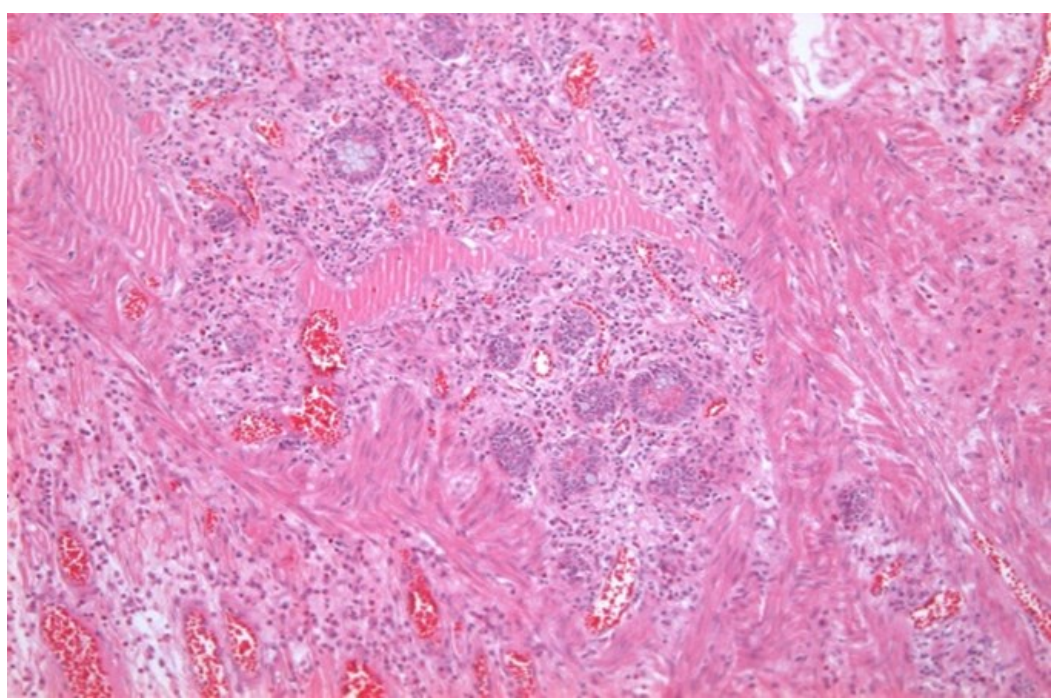

Figure 4a: A few islands of benign glans protruding through the muscularis layer (Magnification $\mathrm{x} 10$ )

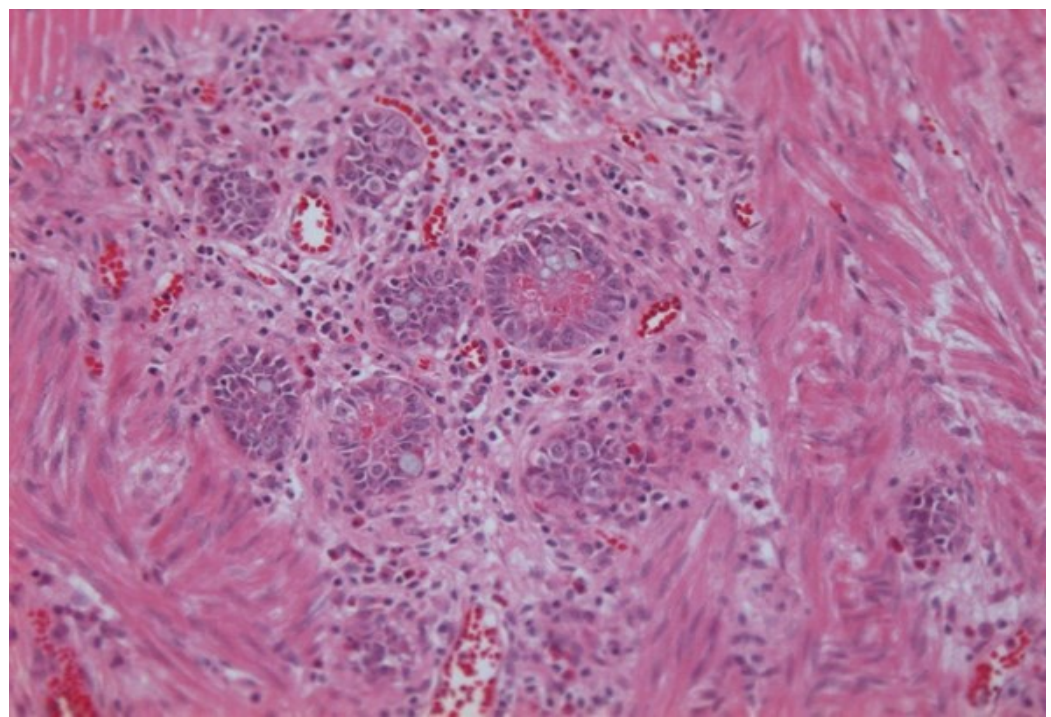

Figure 4b: A few islands of benign glans protruding through the muscularis layer (Magnification $\times 20$ )

\section{DISCUSSION}

Incisional hernia is a post-operative complication that has an incidence of 1 in 5 of abdominal surgery. ${ }^{2}$ Demographic risk factors are age more than 45 years and male gender, ${ }^{2}$ both are present in our patient. Other risk factors are haemoglobin of < $100 \mathrm{~g} / \mathrm{l}$, body mass index $>25$, recurrent incision and previous laparotomy, catecholamine therapy and disturbed wound healing. ${ }^{2}$

Common complications are intestinal obstruction, strangulation and perforation. ${ }^{1}$ The presence of Meckel's diverticulum in an incisional hernia sac is rare and only a few cases have been reported in literature. Perforation is known to occur either due to the increased intraluminal pressure within the entrapped knuckle of bowel or due to the compromised vascular supply to the affected segment of bowel. $^{3}$ As Meckel's diverticulum has ectopic gastric mucosa it may also perforate due to peptic ulceration. ${ }^{1}$
Diagnosis of incisional hernia is predominantly a clinical one although ultrasound is often used to determine the size of the fascial defect and possible content. However in cases of suspected strangulation, a computed tomography would be a better choice for evaluation. An abdominal radiograph would be able to demonstrate dilated bowels in case of intestinal obstruction and pneumoperitoneum in the presence of perforation. ${ }^{4}$ Despite all this diagnostic adjuncts the most accurate diagnosis is made intra-operatively. Thus the rule of thumb is to take the patient to operating theatre as soon as possible in an emergent situation but with adequate pre-operative resuscitation.

Meckel's diverticulum that only becomes symptomatic in less than $4 \%$ of population has a male predominance of 2.5:1. ${ }^{1}$ However there are many other studies quoting up to $5: 1^{1}$ Gastrointestinal bleeding predominates in the paediatric population. 
Diverticulitis is found to be more common in adult men and intestinal obstruction is noted to be more common in females. ${ }^{1}$ It is rare for a Meckel's diverticulum to be entrapped in a hernial sac, moreover in an incisional hernia. Management of the diverticulum depends on the viability of the bowel. A diverticulectomy is sufficient if the bowel is healthy but a segmental resection is indicated in the presence of a non-viable bowel. ${ }^{4}$ For the repair of the fascial defect, we have the options between primary repair or mesh repair however that's beyond our scope of discussion.

\section{CONCLUSION}

In order to manage this type of a rare case, a high index of suspicion is needed to make a clinical diagnosis and to take the patient for surgery without an absolutely confirmed diagnosis. Radiological adjuncts are useful to assist in preoperative planning but should not impede the succession to theatre. Nothing is superior to holding the pathology in your hands rather than playing a guessing game.

\section{REFERENCES}

1. Chen JJ, Lee HC, Yeung CY et al. Meckel's diverticulum: factors associated with clinical manifestations. ISRN Gastroenterol 2014.

2. Höer J, Lawong G, Klinge U, Schumpelick V. Factors influencing the development of incisional hernia. A retrospective study of 2,983 laparotomy patients over a period of 10 years. Der Chirurg 2002; 73: 474-80

3. Jacob TJ, Gaikwad P, Tirkey AJ et al. Perforated obturator Littre hernia. Can J Surg 2009;52:E77.

4. Sharma RK and Jain VK. Emergency surgery for Meckel's diverticulum. World J Emerg Surg 2008; 3:1. 